\title{
MOLECULAR EPIDEMIOLOGY APPROACH TO TACKLE TREATMENT FAILURE IN DRUG RESISTANT TUBERCULOSIS (DR-TB)
}

DOI: 10.36740/WLek202103219

\author{
Mariia G. Dolynska, Gennadii A. Dolynskyi, Tetiana V. Duhlii, Vasyl I. Petrenko \\ BOGOMOLETS NATIONAL MEDICAL UNIVERSITY, KYIV, UKRAINE
}

\begin{abstract}
The aim: To analyze molecular epidemiology features of $M$. tuberculosis in Kyiv oblast; and to identify the safest mode of TB treatment, which will allow clinicians to minimize the risk of drug-resistant strains nosocomial transmission.

Materials and methods: 55 isolates of M. tuberculosis were collected in January-April, 2018 from 31 patients with new cases and 24 patients with re-treatment cases of sputum culture-positive pulmonary TB, in Kyiv oblast, Ukraine. DNA samples extracted from all the isolates were used for 15-loci MIRU-VNTR molecular typing with further M. tuberculosis strains comparison by means of MIRU-VNTRplus web tool (http://www.miru-vntrplus.org).

Results: Phylogenetic tree that reflects strains interrelationship reveals four main clusters, the largest of which spans 34 isolates. The presence of two big subclusters with 10 and 7 identical genotypes inside the largest cluster strongly suggests their tight epidemiologic relationship. Smaller clusters consist of five (Harlem), three (URAL), and two unidentified isolates. 10 singletons were detected, among which LAM, URAL and Cameroon lineages were identified; in these cases, epidemiological connection was presumably ruled out. Conclusions: High clustering level of isolates of $M$. tuberculosis suggests possible contact between patients from whom these isolates were obtained. Predominance of Beijing family in the clusters is associated with high DR-TB level, at least in eastern European countries. Similarity of the clusters isolated from different patients in a household or a hospital suggests high probability of recent disease transmission. Clustering genotypes from households and hospital wards can be a surrogate criterion of infection control effectiveness.
\end{abstract}

KEY WORDS: Mycobacterium tuberculosis, drug resistance, MIRU-VNTR, tuberculosis

Wiad Lek. 2021;74(3 p.ll):669-673

\section{INTRODUCTION}

Drug resistance critically jeopardizes outcomes of tuberculosis (TB) treatment. As of 2020, a treatment success rate in drug-resistant tuberculosis cases (DR-TB) was $57 \%$ globally and 59\% in the European region. TB is the 10th leading cause of death worldwide and, since 2007, it has been the leading cause of death from a single infectious agent, ranking above HIV/AIDS cases. Among 30 countries of high tuberculosis burden, which account $87 \%$ of global DR-TB cases, nine are located in eastern European region, and Ukraine is among them. Among 11,210 patients who started treatment for DR-TB globally, and were also resistant to fluoroquinolones, only $47 \%$ completed treatment successfully and $24 \%$ died; treatment failed for $11 \%$, and $18 \%$ were lost to follow-up or their treatment outcome was not evaluated. India, the Russian Federation and Ukraine accounted for $73 \%$ of this cohort of patients [1]. Thus, DR-TB is a significant public health threat that is of particular importance in Ukraine.

Currently outpatient TB treatment is recognized as a preferred model, which is the safest and the most patient-oriented [2]. Long hospital stay does not improve treatment success [3], but does increase the potential for exposure to unrecognized or inadequately-treated DRTB. From 2014 to 2018, 14 of the 15 eastern European and central Asia (EECA) countries reduced the number of bed days per patient. The relative size of the reduction (which is influenced by both the percentage of TB patients hospitalized and the average length of stay if hospitalized) ranged from $11 \%$ in Romania to $75 \%$ (54 to 14 days) in Armenia. Ukraine is an exception with the average number of days in hospital increase by $12 \%$ in 2017 , remained stable in 2018 [4]. As of 2020, Ukraine reported an average of 85.6 days per patient [5].

This risk is especially high in the cases when M. tuberculosis belongs to Beijing genotype, which is known to be associated with a higher transmission rate [6]. The molecular epidemiology data on M. tuberculosis strains circulating in Ukraine are scarce. Studies performed in southern and eastern Ukraine suggest an increasing prevalence of Beijing family, which was identified in $30.5 \%$ and $54.8 \%$ of new and retreatment cases respectively in a sample from Odesa and Mykolaiv oblast in 2007 [7], and in $81 \%$ of the cases from Kharkiv oblast in 2018 [8]. Whereas the abovementioned regions are located at the transport routes connecting Asia and Europe that can partly explain high prevalence of an Asia-originated Beijing strain [9], TB molecular epidemiology in the central and western parts of Ukraine remains unclear. 


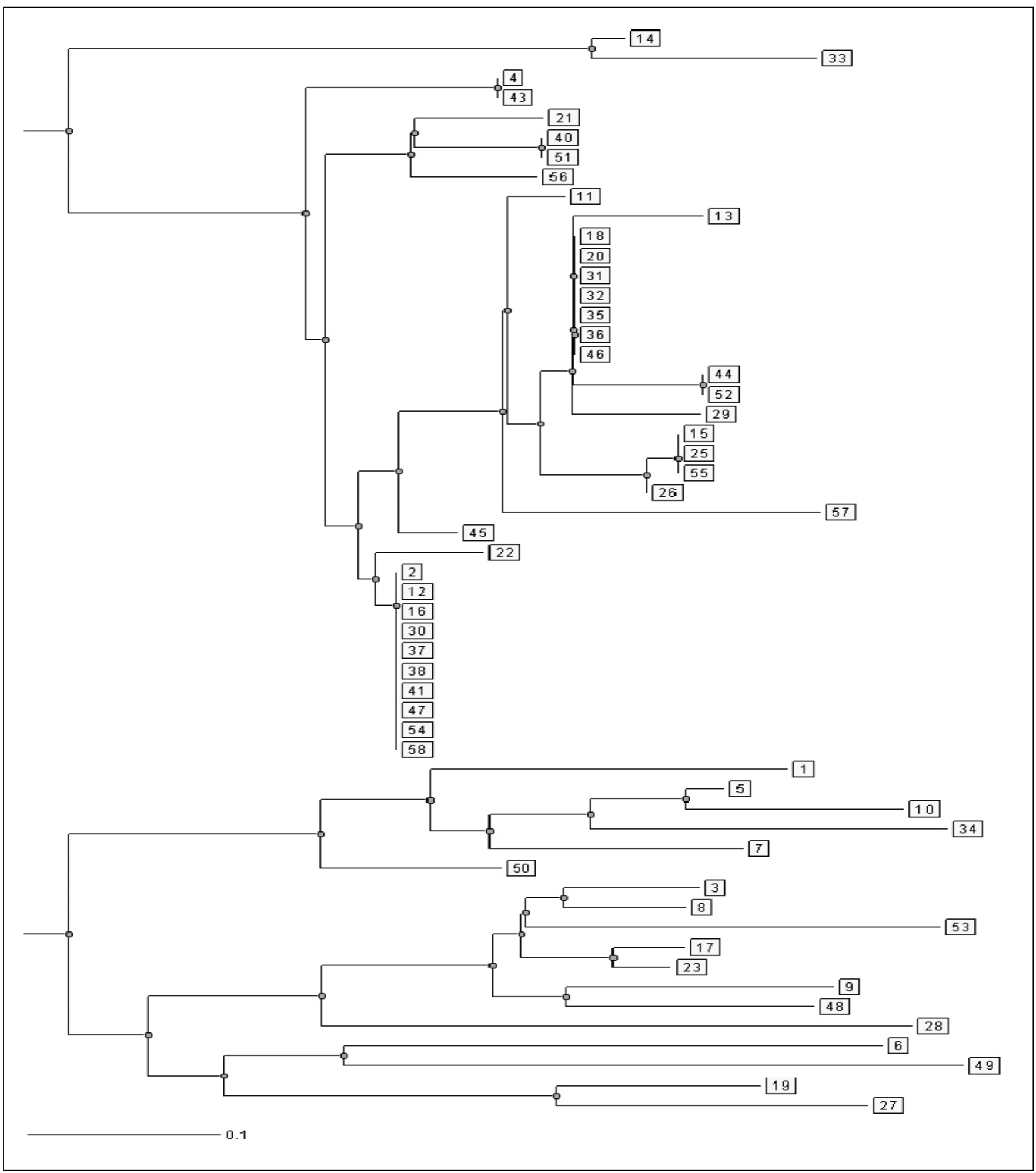

Fig. 1. Dendrogram of annalysed DNA samples interrelationship according to MIRU-VNTR typing

\section{THE AIM}

We hypothesized that patients in hospital for TB treatment are being exposed to and infected with DR-TB. Given that the city of Kyiv as a capital of Ukraine, and Kyiv oblast are experiencing the most active migration in the country, we presumed that Beijing family prevalence in this region can be also high, and therefore the risk of nosocomial TB and DR-TB transmission is significant.

The study aim was (i) to preliminary analyze molecular epidemiology features of M. tuberculosis in Kyiv oblast; and (ii) to identify the safest mode of TB treatment, which will allow clinicians to minimize the risk of drug-resistant strains nosocomial transmission. 
Table I. General statistic results of bioinformatics analysis for MIRU-VNTR imprinting

\begin{tabular}{|c|c|c|c|c|c|c|c|c|c|}
\hline \multicolumn{4}{|c|}{ Samples number } & \multicolumn{6}{|c|}{55} \\
\hline \multicolumn{4}{|c|}{ Clustering level } & \multicolumn{6}{|c|}{$38 \%$} \\
\hline \multicolumn{10}{|c|}{ Alleles diversity } \\
\hline 0424 & 0,48 & 0802 & 0,38 & 1955 & 0,61 & 2401 & 0,31 & 3690 & 0,31 \\
\hline 0577 & 0,40 & 0960 & 0,47 & $2163 b$ & 0,59 & 2996 & 0,55 & 4052 & 0,72 \\
\hline 0580 & 0,02 & 1644 & 0,25 & 2165 & 054, & 3192 & 0,61 & 4156 & 0,29 \\
\hline \multicolumn{10}{|c|}{ Assignment of identified isolates } \\
\hline \multirow{2}{*}{\multicolumn{2}{|c|}{ Beijing }} & \multicolumn{2}{|c|}{ Unknown } & \multicolumn{2}{|c|}{ Haarlem } & \multicolumn{2}{|c|}{ URAL } & \multicolumn{2}{|c|}{ LAM } \\
\hline & $61,82 \%$ & \multicolumn{2}{|c|}{$25,45 \%$} & \multicolumn{2}{|c|}{$7,27 \%$} & \multicolumn{2}{|c|}{$3,64 \%$} & \multicolumn{2}{|c|}{$1,82 \%$} \\
\hline
\end{tabular}

\section{MATERIALS AND METHODS}

55 isolates of $M$. tuberculosis were collected in January-April, 2018 from 31 patients with new cases and 24 patients with re-treatment cases of sputum culture-positive pulmonary tuberculosis. All patients were the residents of Kyiv oblast. 45 (84\%) of the patients had DR-TB. All patients had a history of hospitalisation in TB clinic of at least 3 weeks, and sputum to isolate DNA was collected during the treatment monitoring procedures, normally after one month of treatment for DR-TB casves and after two months for drug-susceptible cases. Patients' age ranged from 17 to 79 years old (median 47 ), with $84 \%$ male and $16 \%$ female.

M. tuberculosis genome typing technique. Molecular epidemiology monitoring was performed using Mycobacterial Interspersed Repetitive Unit-Variable Number of Tandem Repeat (MIRU-VNTR) typing. The method distinguishes the $M$. tuberculosis strains by the difference in the number of copies of tandem repeats at specific regions of the $M$. $t u$ berculosis genome and so enables fast and high-resolution genotyping of $M$. tuberculosis isolates [10]. Polymerase chain reaction (PCR) utilizing forward and reverse primers tailored for each MIRU-VNTR loci results in production of corresponding PCR-amplicons. As the size of a DNA base pairs of each PCR-amplicon is the sum of the size of the tandem repeat plus the offsets at both ends, the number of copies of tandem repeats can be calculated according to the following formula:

Amplicon Size $=$ Offset Size $+($ Copynumber $\times$ Repeat Size $)$ Copynumber $=($ Amplicon Size - Offset Size $) /$ Repeat Size

M. tuberculosis DNA sampling and handling. DNA samples were isolated by CTAB-technique [11] from solid media cultures of mycobacteria obtained from TB patients and stored under refrigeration at $21^{\circ} \mathrm{C}$ until genotyping procedure, which was performed at GenoScreen Innovative Genomics research centre (GenoScreen Corporation, Lille, France). For PCR-amplification core set of 15 VNTRs was selected (MIRU-VNTR loci: 0424, 0577, 0580, 0802, 0960, 1644, 1955, 2163b, 2165, 2401, 2996, 3192, 3690, 4052, 4156) that are both sufficiently and complementary discriminatory [12]. All samples were treated to amplify these 15 markers using 3 quadruplex and 3 simplex PCRs with fluorescent primers specific for the flanking regions of the targeted loci. Amplified fragments were separated by capillary electrophoresis to determine the PCR product sizes.
In case of failure or double alleles, analysis was repeated for corresponding markers using simplex PCRs. As the length of the repeat units is known, sizes reflect the numbers of repeated sequences in the amplified loci. The final result is a portable numerical genotype, corresponding to the repeat number in each locus.

Genotyping datamation. Further bioinformatics analysis was performed by means of MIRU-VNTRplus web tool (http://www.miru-vntrplus.org) for polyphasic genotyping of $M$. tuberculosis complex bacteria, which comprise a reference database containing genotyping data of 186 strains representing the major MTBC lineages [13]. The comparison with the reference database allows identification of a phylogenetic lineage of the analysed strains.

\section{RESULTS AND DISCUSSION}

Statistical data analysis for 55 processed DNA samples presented in Table I.

Phylogenetic tree that reflects strains interrelationship reveals four main clusters, the largest of which spans 34 isolates (Fig. 1). The presence of two big subclusters with 10 and 7 identical genotypes inside the largest cluster strongly suggests tight epidemiologic relationship. Smaller clusters consisted of five (Harlem), three (URAL), and two unidentified isolates. A total of 10 singletons were detected, among which LAM, URAL and Cameroon lineages were identified; in these cases, epidemiological relationship was presumably ruled out.

Among 55 patients whose genotypes were analysed, 34 (62\%), were identified as Beijing cases and had close epidemiological relations, more likely due to contracting the infection from one source. This suggests that Beijing family is a dominating genotype of M. tuberculosis in Kyiv oblast among new and re-treatment cases. As a result, high risk of tuberculosis transmission in hospitals and households arises. In addition, studies from eastern Europe present high association of Beijing genotype and risk of DR-TB [14] in contrast with some studies from Asia [15]. Therefore, prevention interventions become crucial in Ukraine due to high DR-TB burden and risk for contact persons to contract a DR-TB strain, which requires longer and more toxic treatment with poorer results.

This risk increases a need for meticulous contact tracing of each bacteriologically confirmed TB patient, which 
optimally should cover not only household contacts, but also close contacts, i.e. those who share an enclosed space with the index case, such as a place of social gathering, workplace or facility, for extended time periods within the day during 3 months before commencement of the current treatment episode [16].

TB hospitals are the possible place of the DR-TB strains exchange. Given that the majority of TB facilities in Ukraine were built in 1960s and do not meet current infection control standards, this risk is significant. Molecular epidemiology study performed in Latvia where TB hospitals are of the similar time of construction and layouts, demonstrated high association of DR-TB with Beijing genotype (OR 41.67), previous hospitalisation (OR 18.33) and previous TB treatment (OR 17.68). Another finding of this study is that direct epidemiological links in hospitals were found for almost one third (32\%) of DR-TB Beijing cases [14].

Thus, Ukraine urgently needs the paradigm shift with the priority to outpatient treatment in line with the current WHO recommendations. This is the only way to prevent TB and DR-TB transmissions in hospitals. New all-oral DR-TB treatment regimens, which WHO introduced in 2020 [2], enable convenient outpatient treatment without injections for both drug-susceptible and DR-TB cases from the first days of commencement. As it is known that patients who receive TB treatment regimen, which is tailored according to the individual drug resistance patterns, stop spreading the disease after 2-3 weeks of treatment [17], outpatient TB treatment is safe. However, patients with treatment failure can spread the disease and be a source of infections in hospitals and households. This is the reason why early treatment start and meticulous laboratory monitoring are regarded as infection control measures and the prerequisite of a safe treatment mode [18].

MIRU-VNTR typing as a relatively cheap and easyto-perform method can be discriminative enough surrogate of whole genome sequencing (WGS) to follow the disease transmission in households and hospitals, as WGS requires much more investments and experienced personnel $[19,20]$. Therefore, freezing of a culture at the treatment start to ensure the possibility of MIRU-VNTR genotyping in a case of poor treatment response might be a solution to differentiate a superinfection with other genotype of $M$. tuberculosis during the treatment course, from a case of treatment failure. Comparison of the MIRU-VNTR results for the initial and the most recent strain can confirm superinfection if the genotypes are different, and treatment failure in a case of the genetic similarity. In addition, clustering of genotypes obtained from patients with confirmed superinfection who have stayed in same wards is a sign of poor infection control and nosocomial transmission.

\section{CONCLUSIONS}

High clustering level of isolates of $M$. tuberculosis suggests possible close contact among patients from whom these isolates were obtained. Predominance of Beijing family in the clusters is associated with high DR-TB level, at least in eastern European countries. Similarity of the clusters isolated from different patients in a household or a hospital suggests high probability of recent disease transmission. Clustering genotypes from households and hospital wards can be a surrogate criterion of infection control effectiveness.

\section{REFERENCES}

1. WHO. Global tuberculosis report 2020. (WHO/HTM/TB/2020).

2. WHO consolidated guidelines on tuberculosis: Module 4: Treatment: Drug-resistant tuberculosis treatment. WHO. 2020.

3. Tuberculyoz v Ukraini. Analitychno-statystychnyi dovidnyk za 2019 r. Centr Hromadskogo zdorov'a M0Z Ukrainy. 2020 (in Ukrainian). https://phc.org.ua/sites/default/files/users/user90/TB_surveillance_ statistical-information_2019 dovidnyk.pdf.

4. WHO. Global tuberculosis report 2019 (WHO/HTM/TB/2019).

5. WHO. Global tuberculosis report 2018 (WHO/HTM/TB/2018).

6. Liu Y., Zhang X., Zhang Y. et al. Characterization of Mycobacterium tuberculosis strains in Beijing, China: drug susceptibility phenotypes and Beijing genotype family transmission. BMC Infect Dis. 2018;18:658. doi: 10.1186/s12879-018-3578-7.

7. Nikolayevskyy V.V., Brown T.J., Bazhora Y.I. et al. Molecular epidemiology and prevalence of mutations conferring rifampicin and isoniazid resistance in Mycobacterium tuberculosis strains from the southern Ukraine. Clin Microbio Infect. 2007;13(2):129-138. doi: 10.1111/j.14690691.2006.01583.x.

8. Daum L.T., Konstantynovska 0.S., Solodiankin 0.S. et al. Nextgeneration sequencing for characterizing drug resistance-conferring Mycobacterium tuberculosis genes from clinical isolates in the Ukraine. J Clin Microbiol. 2018;56(6):e00009-18. doi: 10.1128/JCM.00009-18.

9. PichatC.,Couvin D.,CarretG. etal.Combined genotypic, phylogenetic, and epidemiologic analyses of Mycobacterium tuberculosis genetic diversity in the Rhône Alpes region, France. PLoS One. 2016;11(4):e0153580. doi: 10.1371/journal.pone. 015358029-138.

10. Skenders G., van Soolingen D. Revised by Niemann S. and Nikolayevskyy V. Molecular typing of Mycobacterium tuberculosis complex isolates. In: European Centre for Disease Prevention and Control. Handbook on TB laboratory diagnostic methods for the European Union, Stockholm: ECDC. 2016: 83-91.

11. van Soolingen D., de Haas P.E., Hermans P.W., van Embden J.D. DNA fingerprinting of Mycobacterium tuberculosis. Methods Enzymol. 1994;235:196-205. doi: 10.1016/0076-6879(94)35141-4.

12. Supply P., Allix C., Lesjean S., et al. Proposal for standardization of optimized mycobacterial interspersed repetitive unit-variable-number tandem repeat typing of Mycobacterium tuberculosis. J Clin Microbiol. 2006;44(12):4498-4510. doi: 10.1128/JCM.01392-06.

13. Weniger T., Krawczyk J., Supply P. et al. MIRU-VNTRplus: a web tool for polyphasic genotyping of Mycobacterium tuberculosis complex bacteria. Nucleic Acids Res. 2010;38:326-331. doi: 10.1093/nar/gkq351.

14. Nodieva A., Jansone I., Broka L. et al. Recent nosocomial transmission and genotypes of multidrug-resistant Mycobacterium tuberculosis. Int J Tuberc Lung Dis. 2010;14(4):427-33.

15. Liu Y., Jiang X., LiW. et al. The study on the association between Beijing genotype family and drug susceptibility phenotypes of Mycobacterium tuberculosis in Beijing. Sci Rep. 2017;7:15076. doi: 10.1038/s41598017-14119-z. 
16. Fair E., Miller C.R., Ottmani S.E. et al. Tuberculosis contact investigation in low- and middle-income countries: standardized definitions and indicators. Int J Tuberc Lung Dis. 2015;19(3):269-72. doi: 10.5588/ ijtld.14.0512.

17. Dharmadhikari A.S., Mphahlele M., Venter K. et al. Rapid impact of effective treatment on transmission of multidrug-resistant tuberculosis. Int JTuberc Lung Dis. 2014;18(9):1019-1025. doi: 10.5588/ ijtld.13.0834.

18. Van Cutsem G., Isaakidis P., Farley J. et al. Infection control for drugresistant tuberculosis: early diagnosis and treatment is the key: Table 1. Clin Infect Dis. 2016;62:238-243. doi: 10.1093/cid/ciw012.

19. Rasoahanitralisoa R., Rakotosamimanana N., Stucki D. et al. Evaluation of spoligotyping, SNPs and customised MIRU-VNTR combination for genotyping Mycobacterium tuberculosis clinical isolates in Madagascar. PLoSOne. 2017;12(10):e0186088. doi: 10.1371/journal.pone.0186088.

20. Liu Y., Zhang X., Zhang Y. et al. Characterization of Mycobacterium tuberculosis strains in Beijing, China: drug susceptibility phenotypes and Beijing genotype family transmission. BMC Infect Dis. 2018;18(1):658. doi: 10.1186/s12879-018-3578-7.
Research programme "Use of novel technologies for tuberculosis diagnosis and treatment" of Ministry of health of Ukraine (2018-2020, № state registration 0118U001213).

\section{ORCID and contributionship:}

Mariia G. Dolynska: 0000-0001-8862-1554 A,B,D

Gennadii A. Dolynskyi: 0000-0001-9942-293X B, C, D

Tetiana V. Duhlii: 0000-0003-2071-584X ${ }^{B}$

Vasyl I. Petrenko: 0000-0002-5450-308X A, E, F

\section{Conflict of interest:}

The Authors declare no conflict of interest.

\author{
CORRESPONDING AUTHOR \\ Maria Dolynska \\ Bogomolets National Medical University \\ 68 Avtozavodska st., 02000 Kyiv, Ukraine \\ tel: +380500759513 \\ e-mail:dolynskamaria@gmail.com \\ Received: 18.11 .2020 \\ Accepted: 09.03.2021
}

A - Work concept and design, B - Data collection and analysis, C - Responsibility for statistical analysis,

D-Writing the article, $\mathbf{E}$-Critical review, $\mathbf{F}$ - Final approval of the article 